\title{
E⿵⺆⿻二丨
}

因@(1) $\circledast$

http://pubs.acs.org/journal/aelccp

\section{Physical Passivation of Grain Boundaries and Defects in Perovskite Solar Cells by an Isolating Thin Polymer}

\author{
Efrain Ochoa-Martinez, * Mario Ochoa, Roberto D. Ortuso, Parnian Ferdowsi, Romain Carron, \\ Ayodhya N. Tiwari, Ullrich Steiner, and Michael Saliba*
}

Cite This: ACS Energy Lett. 2021, 6, 2626-2634

Read Online

ACCESS | Llll Metrics \& More | 国 Article Recommendations | st Supporting Information

ABSTRACT: Passivation and interlayer engineering are important approaches to increase the efficiency and stability of perovskite solar cells. Thin insulating dielectric films at the interface between the perovskite and the charge carrier transport layers have been suggested to passivate surface defects. Here, we analyze the effect of depositing poly(methyl methacrylate) (PMMA) from a very low-concentration solution. Spatial- and time-resolved photoluminescence and atomic force microscopy analyses of samples with diverse morphologies demonstrate the preferential deposition of PMMA in topographic depressions of the perovskite layer, such as grain and domain boundaries. This

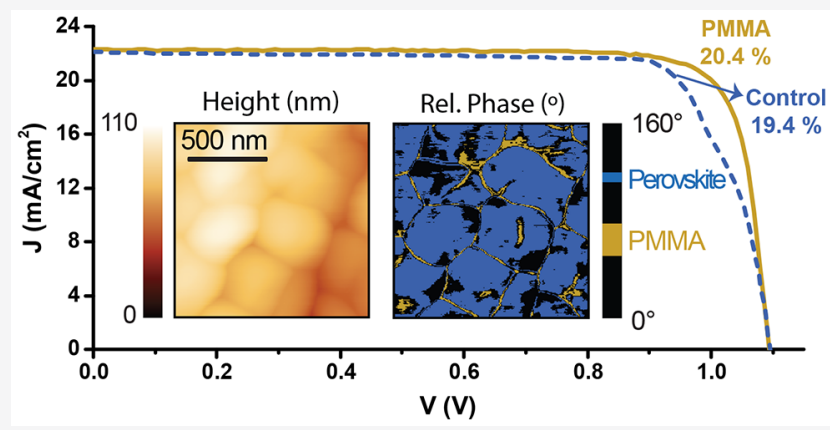
treatment results in an increase in the fill factor of more than $4 \%$ and an absolute efficiency boost exceeding $1 \%$, with a maximum efficiency of $20.4 \%$. Based on these results, we propose a physical isolation mechanism rather than a chemical passivation of perovskite defects, which explains not only the data of this study but also most results found in earlier works.

$\mathrm{R}$ enewable energies have the highest growth rate in installed capacity among all power generation sources during the last decades. Among those, photovoltaic (PV) energy plays and increasingly important role and accounts now for $3 \%$ of the global electricity generation, overtaking wind energy as the fastest growing renewable technology. ${ }^{2,3}$ Silicon photovoltaics constitute by far the highest proportion of the photovoltaic production, ${ }^{4}$ but a significant increase in the installation of photovoltaics is still required to achieve the global objectives for decarbonization. Other technologies, such as thin film and third-generation photovoltaics, whether by themselves or in combination with silicon, might contribute to further increases in device efficiencies and/or reductions in price per watt $(\$ / W p)$. In particular, perovskite solar cells (PSCs) have shown the fastest increase in device efficiency of all the photovoltaic technologies. ${ }^{5}$ Perovskites are polyelemental, structurally complex materials. One of their major advantages over other PV technologies is that they can be deposited through simple solution processing at relatively low temperatures. One manufacturing obstacle of PSCs is the way how perovskite crystallization from solution is initiated; during spin-coating of the perovskite layer from solution, a poor solvent for the perovskite is deposited onto the spinning substrate, causing the perovskite to precipitate from solution, forming a polycrystalline layer. This so-called antisolvent process requires the use of relatively large amounts $\left(\geq 100 \mu \mathrm{L} / \mathrm{cm}^{2}\right)$ of organochlorides or other organic solvents.

Most high-efficiency PSCs, including the current efficiency world record, employ this method. ${ }^{5}$ They contain a compact perovskite multicrystalline layer comprised of submicrometer crystallites, but device areas are typically limited to $\sim 1 \mathrm{~cm}^{2}$, because the antisolvent process is not suitable for the production of large-area devices. An important part of the current research efforts is therefore aiming toward more scalable deposition methods, such as blade-coating ${ }^{6}$ or evaporation deposition. ${ }^{7}$ Alternatively, "flash infrared annealing” (FIRA) is a liquid deposition technique that avoids the use of antisolvents for the manufacture of perovskite layers. Through the use of short pulses of high-power infrared light, it

Received: June 8, 2021

Accepted: June 23, 2021

Published: June 30, 2021 

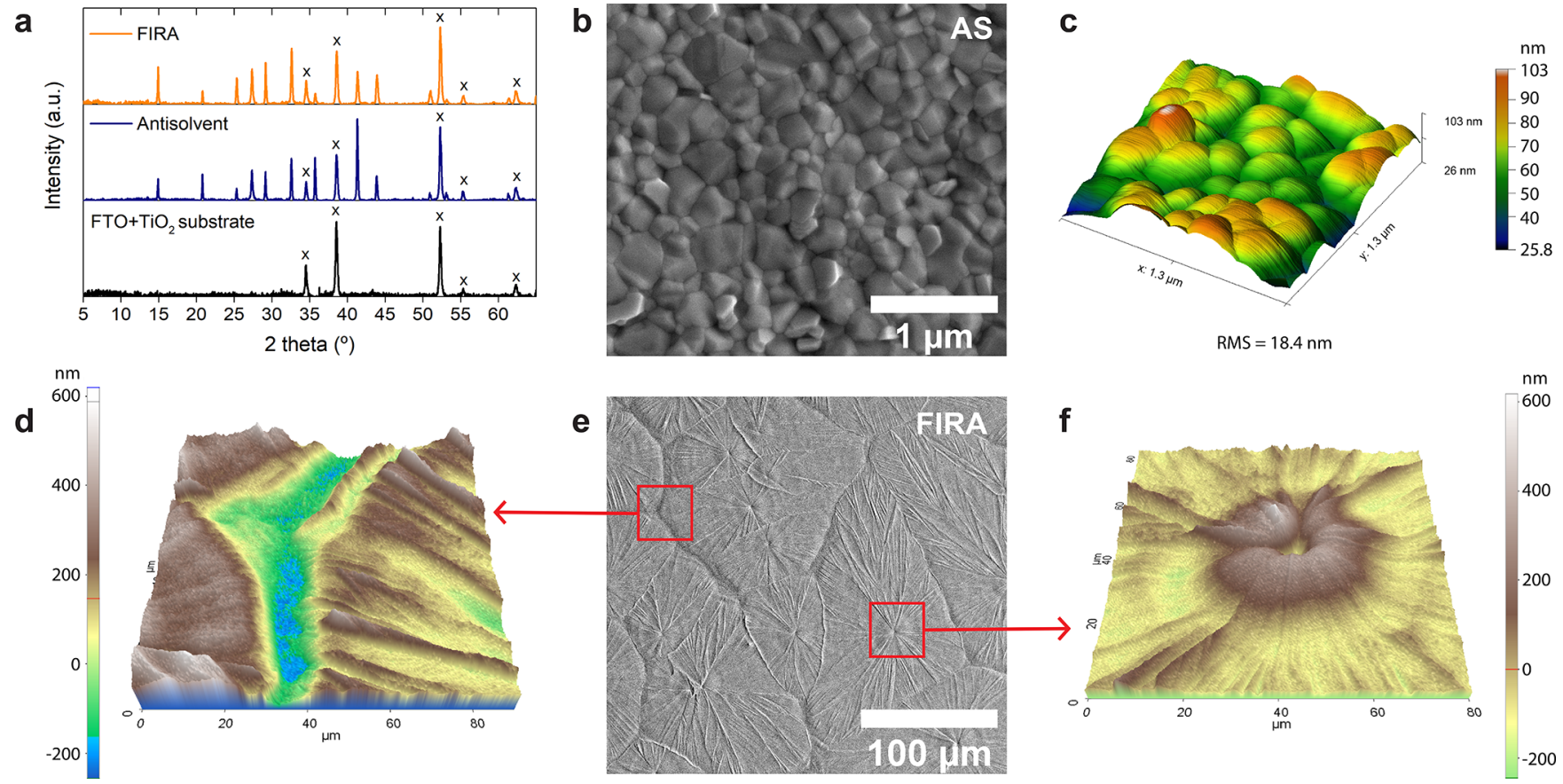

Figure 1. XRD spectra of FTO + $\mathrm{TiO}_{2}$ substrates, bare and covered by antisolvent and FIRA annealed triple-cation perovskite films (a). SEM and AFM images of antisolvent $(b, c)$ and FIRA $(d-f)$ perovskite films.

achieves the crystallization of the perovskite in less than $2 \mathrm{~s}{ }^{8}$ resulting in a compact layer with the same chemical composition and crystal structure as obtained with antisolvent. $^{9,10}$

Despite all manufacturing efforts, all deposition methods result in perovskite films with variable degrees of defects that limit the device performance. They can be present in the form of extended $2 \mathrm{D}$ and $3 \mathrm{D}$ defects such as pinholes and grain boundaries or localized defects like bulk vacancies, excess interstitials, or undercoordinated surface ions that may induce deep trap states for charge carriers. Major improvements in efficiency and stability have been achieved through different defect passivation methods for silicon, ${ }^{11}$ CIGS, ${ }^{12}$ and PSC $^{13}$ photovoltaics, and further improvements in passivation are a key aspect to ensure the high stabilities required in commercial perovskite solar cells ${ }^{14,15}$ and LEDs; ${ }^{16}$ other authors signal passivation methods, like the one discussed in this work, as a significant step toward tandem solar cells with efficiencies exceeding $30 \%$. $^{17}$

Recently, the use of several types of large molecules in the form of Lewis acids and bases were shown to provide effective surface passivation in PSCs, reducing the number of defects and increasing carrier lifetimes. ${ }^{15,18}$ Poly(methyl methacrylate) (PMMA) is such a Lewis base; it has been proposed as a nucleation template to improve crystallization, ${ }^{19}$ a planarizing $^{20,21}$ and passivation agent in PSCs. ${ }^{22-24}$ It has been used between the perovskite and the electron transport layer $(\mathrm{ETL})^{20,21,23}$ and between the perovskite and the hole transport layer (HTL). ${ }^{20,25}$ The combination of PMMA with phenyl- $\mathrm{C}_{61}$-butyric acid methyl ester (PCBM) to improve the conductivity of the interlayer compound has also been studied. $^{20,21,23,24}$

Several authors have proposed theories for the mechanism behind the performance improvement caused by these interlayers. The conformal coating of thin layers over the perovskite and the passivation of surface defects has been proposed. $^{25,26}$ These passivating layers should, however, not prevent the displacement of carriers across the interface to the adjacent transport layer. According to the conformal coating model, the carriers need to tunnel through the dielectric layer, similar to metal-oxide-semiconductor (MOS) devices, but little is known about the morphology and the transport properties of these layers. In addition, the relation between the deposition conditions and the thickness of the layer remains unexplored.

The present study analyzes the deposition of PMMA with a very low-concentration solution onto PSCs produced by both the antisolvent and FIRA techniques. Flash annealing offers the possibility to explore a deposition method that has industrial potential, but mainly the large domain structure produced by FIRA, with features in the order of microns, provides a perfect model for the analysis of the deposition behavior of polymeric layers. It enables the morphology of the deposited polymer and its effect on the device performance to be studied. The characterization of the degree of passivation provided has been assessed through spatially and time-resolved photoluminescence, while a detailed atomic force microscopy analysis, complemented with ellipsometric and electrical measurements, allows distinguishing the PMMA morphology once deposited onto the perovskite film and its effect on finished devices.

Triple-cation perovskite layers with the composition $\mathrm{Cs}_{0.05}\left(\mathrm{FA}_{0.9} \mathrm{MA}_{0.1}\right)_{0.95} \mathrm{~Pb}\left(\mathrm{I}_{0.9} \mathrm{Br}_{0.1}\right)_{3}$ were prepared using FIRA and antisolvent (AS) processes. A cross-sectional image and a schematic drawing of a PSC are shown in Figure S1. Reference PSCs without PMMA were compared to devices with PMMA treatment. Figure 1a shows the presence of the same peaks in the XRD analysis, but the peak intensities differ for the two preparation methods, indicating differences in the perovskite crystal orientation. ${ }^{8,9}$ Then, from the crystallographic point of view, both types of samples are polycrystalline with the same composition but different crystallite sizes and orientations. ${ }^{10}$

While similar in crystallinity, the topography is quite different (Figure 1b,e). Films produced by the AS process 


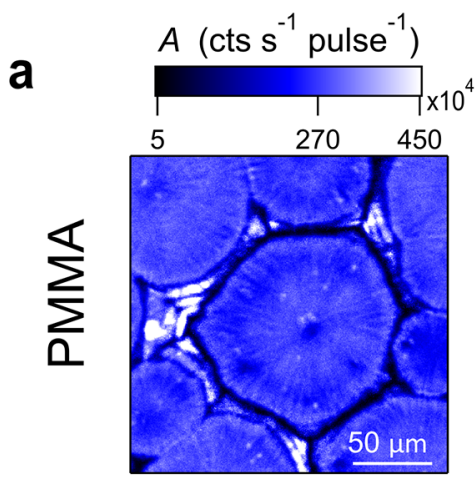

b

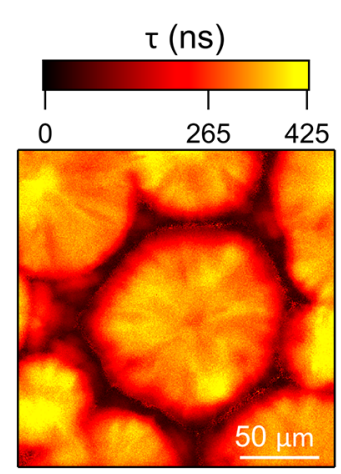

d

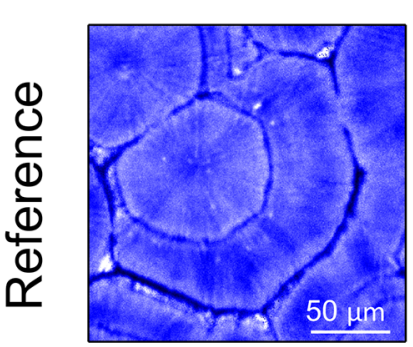

g

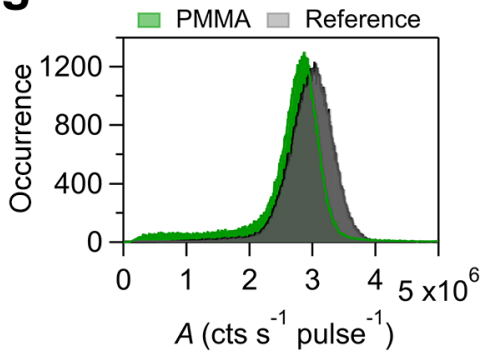

e



h

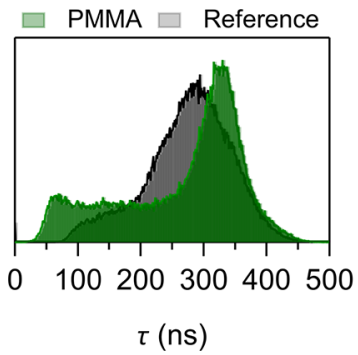

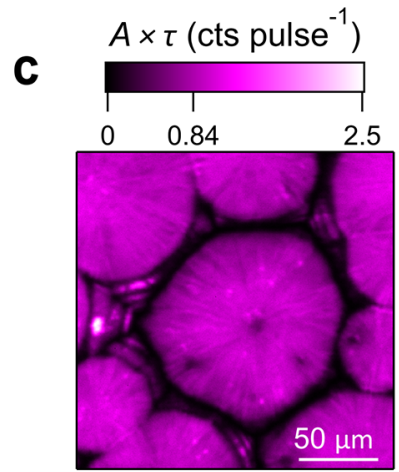

f

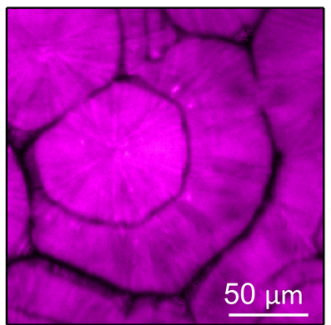

i

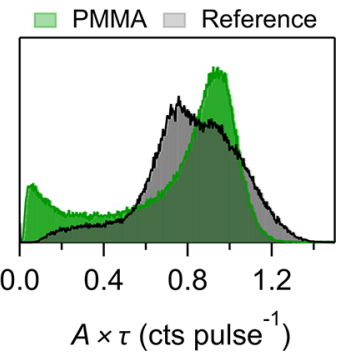

Figure 2. Spatially resolved TRPL mappings of flash annealed triple-cation perovskite films showing intensity $A$, lifetime $\tau$, and $A \times \tau$ for films with $(\mathrm{a}-\mathrm{c})$ and without (d-f) PMMA treatment. Below, histograms of intensities (g), lifetime (h), and lifetime $\times$ intensity (i).

consist in most cases of tightly packed crystallites providing a good surface coverage (Figure $1 \mathrm{~b}$ ). They have an average thickness of the perovskite capping layer of $500 \mathrm{~nm}$, the roughness is in the range of $10-20 \mathrm{~nm}$ (Figure 1c), and grain sizes are in the range of some hundreds of nanometers.

FIRA films, on the other hand, present a similar average thickness, but with a quite characteristic "volcano-like" topography, with differences of several hundreds of nanometers in the center and in the edges of the domains, shown in Figure $1 \mathrm{~d}-\mathrm{f}$, these structures spread across the domains, which have areas in the range of the hundreds of micrometers. During FIRA annealing, a good wetting of the substrate by the precursor solution is crucial, since the crystallization typically forms isolated islands. Only the fast crystallization prevents the dewetting of the film, because solvent evaporation outpaces the material diffusion. ${ }^{27}$ Despite parameter optimization, the perovskite films exhibit small uncovered areas and regions with low film thickness at the domain boundaries. These valleys can reach several micrometers in width (Figure 1d). Even though these are scarce, they offer a path for localized electrical shunting between the electron and hole transport layers. Nevertheless, fast annealing processes for perovskites have been implemented by other groups, ${ }^{28}$ and FIRA devices have already surpassed efficiencies of $20 \%{ }^{29}$

For both techniques, AS and FIRA, the presence of some pinholes, cracks, and other distributed defects is unavoidable, as verified easily by SEM analysis. Localized point and surface defects are more difficult to detect. Aiming to gain further insight into possible passivation effects of thin PMMA layers on the FIRA and antisolvent devices, we analyzed their charge carrier dynamics through spatially and time-resolved photoluminescence (TRPL) spectroscopy.

Figure 2 shows TRPL maps of FIRA perovskite layers with and without PMMA deposition, with an $\mathrm{FTO} / \mathrm{c}-\mathrm{TiO}_{2} / \mathrm{m}$ $\mathrm{TiO}_{2} /$ perovskite/(PMMA) layer structure. Three different maps per device are shown, the amplitude $A$, lifetime $\tau$, and $A \times \tau$. The initial amplitude $A$ is the photoluminescence signal prior to any recombination process. The lifetime characterizes the PL decay, whereas $A \times \tau$ is a qualitative joint measure of the two parameters. In addition, under low-injection conditions, $A \times \tau$ is proportional to the PL yield of the material.

Figure 2 allows the domain structure arising from the FIRA annealing to be discerned, with its volcano-like shape of several tenths to hundreds of micrometers in width for both samples, with and without PMMA. The amplitudes $A$ (Figure 2a,d) resemble the topography of the sample and correlate with the thickness variations of Figure 1d,f. The lifetime maps in Figure 2b,e show similar carrier lifetimes of $\sim 400 \mathrm{~ns}$ within the domains, reducing to $\sim 300$ ns close to the domain boundaries. At the domain boundaries, the lifetimes are $\sim 100$ and $\sim 50 \mathrm{~ns}$ for samples without and with PMMA respectively, which is 
FIRA

a

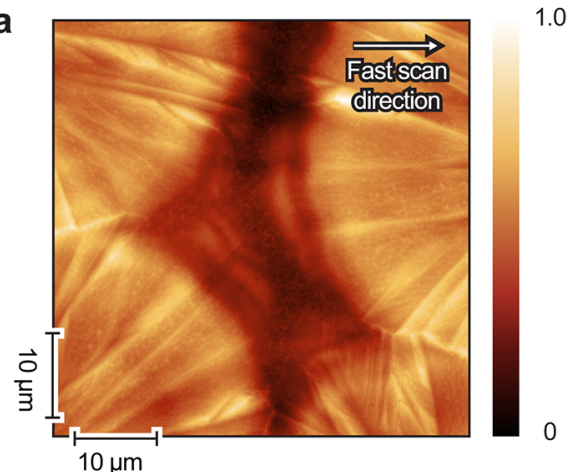

b
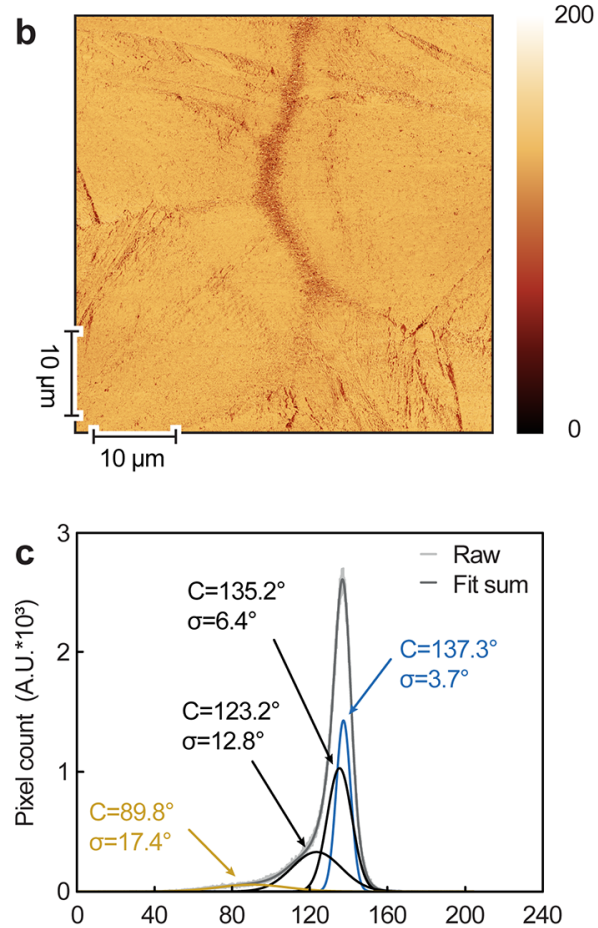

d

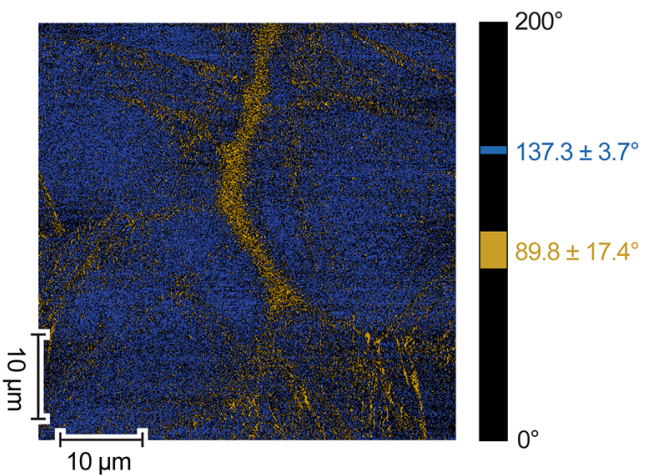

Antisolvent

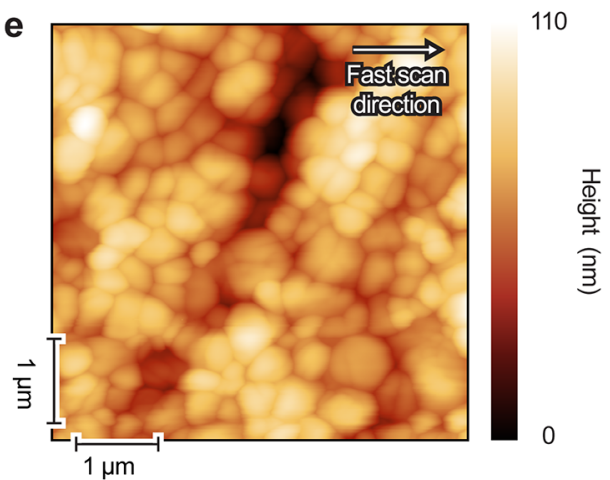

160

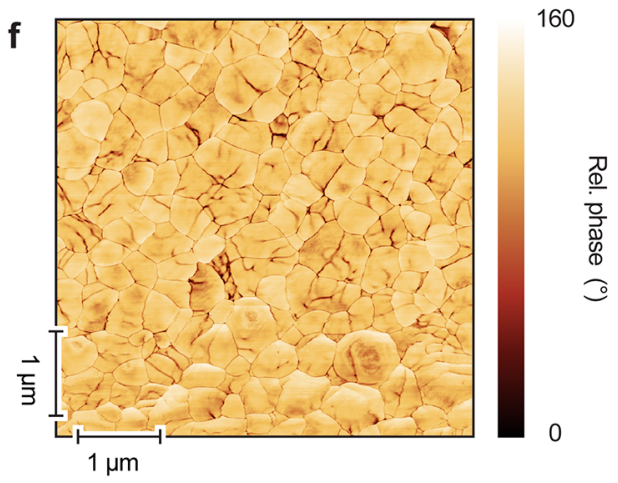

g

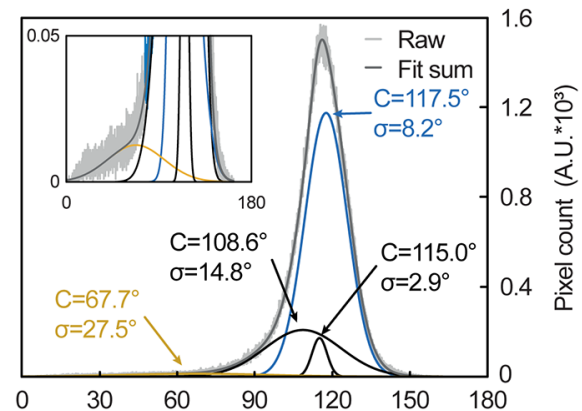

h

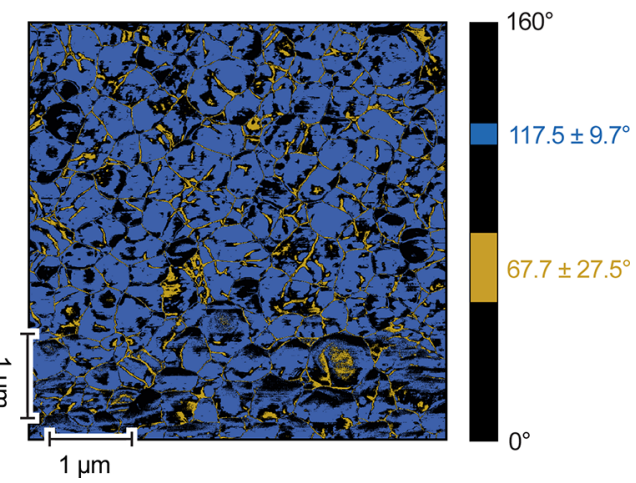

Figure 3. AFM scans showing a domain boundary of FIRA (left) and a full region of antisolvent (right) PMMA-treated triple-cation perovskite films. (a,e) Height image (topography), (b,f) phase channel, (c,g) phase channel histogram and deconvolution analysis, (d,h) segmented color-coded image of the phase channel with two selected phase regions for each sample, the phase signal outside these two regions is color-coded black.

about 4 and 8 times lower compared to the domain centers. Figure S2 shows decay curves for the three different domain regions. The lower PL signal at the domain boundaries is also evident from the histograms in Figure $2 g-i$. These histograms reveal $\tau$ values well below 100 ns for PMMA-covered samples, which do not appear in samples without PMMA. This is consistent with the decays shown in Figure S2. Similar observations can be drawn for the amplitude $A$, where a long 
tail is observed for PMMA-covered samples at values below 2 $\times 10^{6}$. The combination of the low $A$ and $\tau$ values are magnified in the $A \times \tau$ histogram, with both parameters contributing similarly to the $A \times \tau$ maps. Note that the lowsignal regimes at the domain boundaries in Figure 2 are wider for the PMMA-covered samples with respect to the reference.

The difference in lifetime indicates structural or composition differences within the domain. The domains in FIRA films are composed of a collection of grains with different sizes and preferential orientations. ${ }^{10,27}$ This crystalline texture affects the quality, charge transport properties, and trap density of the material. Minor variations in the PL signals stemming from the interior of the domain are discernible in all samples; the comparison of different maps (not shown) show that there is no particular trend among the sample-to-sample variations. In particular, no significant differences between PMMA-treated and reference samples were detected.

On the other hand, at the domain boundaries, the large lifetime and PL intensity decrease for both PMMA and reference can be ascribed mainly to a reduced amount of material compared to the interior of the domains. This is supported by the AFM measurements in Figure 1d,f, showing height differences between the center of the domain and the edges of up to $1 \mu \mathrm{m}$. A lower PL signal for thinner regions is expected because first of an incomplete light absorption ${ }^{30}$ and second of the increased relative influence of recombination at the front and back surface for thinner absorbers with a negative impact on the charge carrier lifetime. In the same way, the wider region of low PL signal and carrier lifetime at the domain boundaries for PMMA-treated samples could also be associated with reduced absorber thickness, rather than caused by PMMA deposition. Nevertheless, this can be ruled out by analyzing a series of time-gated PL maps (Figure S3a,b) and corresponding line profiles (Figure $\mathrm{S} 3 \mathrm{c}, \mathrm{d}$ ) along representative domain boundaries, confirming that the lower PL signal close to the domain boundaries of PMMA-treated samples is not related to a different perovskite thickness.

These results are consistent with those by Chen et al., ${ }^{21}$ who measured lower (not spatially resolved) PL signals after PMMA deposition, attributed to a diminished effect of interface traps and charge accumulation. The results of Figure 2 show significantly lower lifetimes arising only from the domain boundaries. Indeed, considering the analysis of Krogmeier et al., ${ }^{29}$ it is reasonable to attribute a passivation mechanism to PMMA despite of the lower measured carrier lifetime but only at the domain boundaries (Figure S2).

TRPL maps were also acquired for perovskite films made by the antisolvent method (see Figure S4). The spatial resolution of TRPL is too low to resolve the grain structure of these samples. The impact of a PMMA treatment on the TRPL maps is not evident. Nevertheless, the histograms in Figure S4 suggest a minor reduction in both intensity and lifetime after the PMMA treatment.

Atomic force microscopy (AFM) was used with both types of samples to visualize the location of the PMMA. When AFM is used in amplitude modulated (tapping) mode, monitoring the oscillation phase offset allows detecting changes in the tipsample interaction, alongside the acquisition of the sample topography. A 2D phase map, therefore, provides a map of different materials that are present on the surface. ${ }^{31}$ In this analysis, the phase information allows the distribution of PMMA on the perovskite surface to be localized.
Figure 3a shows the results of an AFM analysis of a FIRA sample after the PMMA deposition, focusing on a domain boundary. The height image in Figure $3 \mathrm{a}$ shows a reduced perovskite thickness at the domain boundary, by about $1 \mu \mathrm{m}$, which could provide shunting pathways between the ETL below the perovskite and the HTL on top of it. Figure $3 \mathrm{~b}$ shows the corresponding relative phase map. While overall homogeneous, a notable phase difference is discernible at the domain boundary. Smaller phase differences coincide with some of the perovskite topographic domain stripes, in addition to some isolated dark spots.

Figure $3 c$ shows the histogram of all the relative phase values from Figure $3 \mathrm{~b}$. The main contributions stem from the 123 to $137^{\circ}$ phase region, with the strongest contributions at 135 and $137^{\circ}$ with a low variance. There is however a broad tail indicative of a significantly different phase region centered at $90^{\circ}$. This enables the reconstruction of the phase image by assigning different colors to these two-phase regions shown in Figure $3 \mathrm{~d}$. This highlights the different nature of the AFM-tip interactions with the sample, showing a contrast between the domain interior and its boundary, in agreement with the TRPL mapping.

A quantitative analysis of the phase image is difficult; nevertheless, Figure $3 \mathrm{~d}$ shows that a robust material contrast can be obtained. A similar analysis performed in a sample without PMMA treatment, shown in Figure S5, is instructive. While the sample topography in Figure S5a is very similar to that of Figure $3 \mathrm{a}$, the phase image is almost without contrast (Figure S5b) and does not clearly highlight the net difference in regions as seen for the sample with PMMA. Consequently, the histogram in Figure S5c is close to a normal distribution without the presence of the tail seen in Figure $3 \mathrm{c}$. The phase signal exhibits only one phase region $\left(97-104^{\circ}\right)$, indicating the absence of a material difference between the domain interior and its boundary.

The same analysis was carried out with films deposited using the traditional antisolvent method. Figure $3 e-h$ shows the results for a sample after PMMA treatment, compared to Figure S6 for a reference sample without PMMA. Despite the already mentioned morphological differences to the FIRA sample, the results of the phase analysis are very similar. A clear contrast is discernible in Figure $3 \mathrm{f}$ of bright grains separated by dark boundaries, indicating the presence of a different material at the grain edges compared to the grains themselves.

The histogram in Figure $3 \mathrm{~g}$ has a less pronounced tail compared to the FIRA devices because of the smaller widths of the intergrain regions. Nevertheless, a different phase with a relative phase value around $68^{\circ}$ is discernible, presenting a clear contrast to the main contribution at $\sim 118^{\circ}$. The colorcoded representation in Figure $3 \mathrm{~h}$ shows clearly that the lower relative phase material is localized mainly at the grain boundaries. The reference sample in Figure S6b shows almost no phase contrast apart from a lower phase region near the center of the image, which may stem from a contamination or a $\mathrm{PbI}_{2}$ grain.

Note that the relative phase differences between the main contribution and the lower phase contribution are similar in Figures $3 \mathrm{~d}$ and $3 \mathrm{~h}\left(\sim 50^{\circ}\right)$. Since the absolute phase values are arbitrary (they depend on the AFM scanning parameters), this phase difference is indicative of a very similar material, which is not observed in samples without PMMA treatment. Since the only difference between the samples with respect to their reference samples is the PMMA addition, is very likely the 

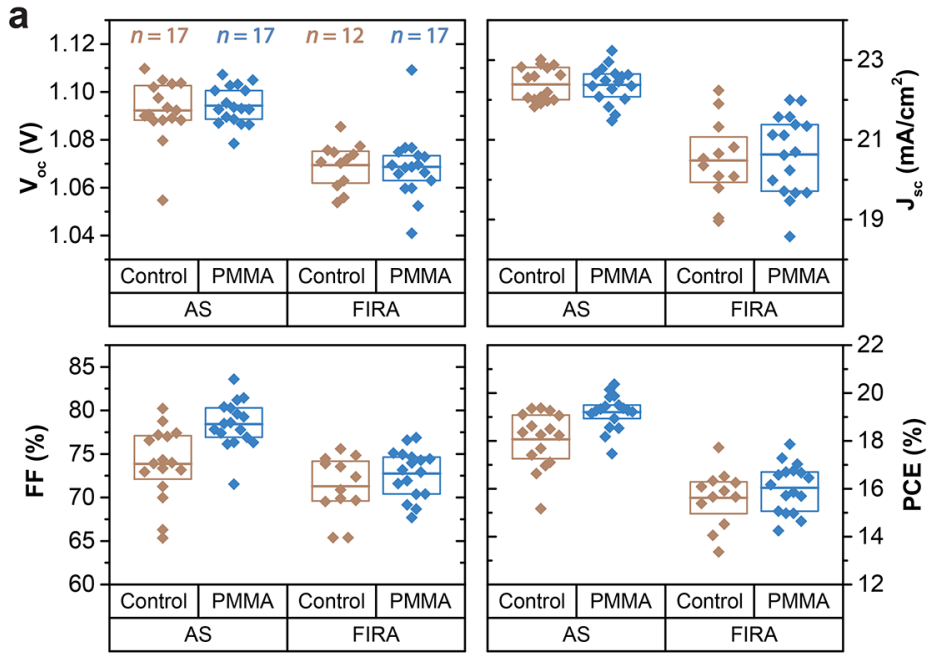

b

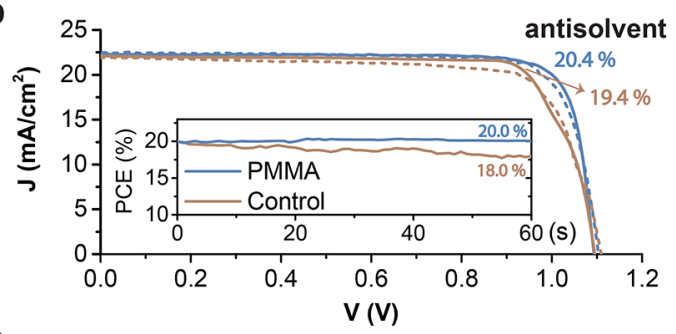

C

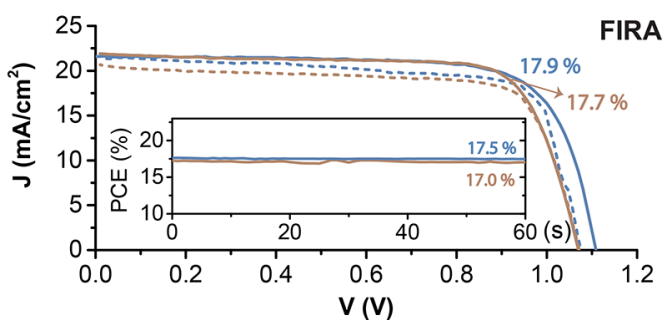

Figure 4. Left: (a) Photovoltaic parameters for reference (brown) and PMMA-treated (blue) AS and FIRA devices. Right: $J-V$ curves for champion devices prepared with (b) the AS and (c) the FIRA process, where full and dashed lines indicate forward and reverse scans, respectively. The insets in $b$ and $c$ show the stabilized power output (SPO) for the AS and FIRA champion devices, respectively, obtained at the maximum power point voltage.

Table 1. Photovoltaic Parameters (Average and Champion Devices) for Reference and PMMA-Treated PSCs

\begin{tabular}{|c|c|c|c|c|c|c|c|}
\hline & & $V_{\mathrm{oc}}(\mathrm{mV})$ & $J_{\mathrm{sc}}\left(\mathrm{mA} \mathrm{cm} \mathrm{cm}^{-2}\right)$ & FF (\%) & PCE (\%) & $R_{\mathrm{sh}}\left(\mathrm{k} \Omega \mathrm{cm}^{2}\right)$ & $R_{\mathrm{s}}\left(\Omega \mathrm{cm}^{2}\right)$ \\
\hline \multirow{2}{*}{ AS + PMMA } & average & $1094 \pm 6$ & $22.4 \pm 0.4$ & $78.4 \pm 2.0$ & $19.2 \pm 0.5$ & $19.8 \pm 4.1$ & $3.6 \pm 0.5$ \\
\hline & champion & 1094 & 22.3 & 83.6 & 20.4 & 32.9 & 2.3 \\
\hline \multirow[t]{2}{*}{ AS } & average & $1092 \pm 9$ & $22.4 \pm 0.4$ & $73.9 \pm 3.1$ & $18.1 \pm 0.9$ & $13.3 \pm 4.3$ & $4.4 \pm 0.7$ \\
\hline & champion & 1093 & 22.1 & 80.2 & 19.4 & 19.6 & 3.1 \\
\hline \multirow[t]{2}{*}{ FIRA + PMMA } & average & $1069 \pm 9$ & $20.6 \pm 0.8$ & $72.8 \pm 2.3$ & $16.0 \pm 0.8$ & $7.0 \pm 2.8$ & $4.7 \pm 1.0$ \\
\hline & champion & 1109 & 21.6 & 74.6 & 17.9 & 7.1 & 4.3 \\
\hline \multirow[t]{2}{*}{ FIRA } & average & $1069 \pm 7$ & $20.5 \pm 0.8$ & $71.3 \pm 2.8$ & $15.6 \pm 0.9$ & $6.4 \pm 2.2$ & $5.4 \pm 0.9$ \\
\hline & champion & 1070 & 21.9 & 75.6 & 17.7 & 9.1 & 4.9 \\
\hline
\end{tabular}

PMMA accumulates in both sample types (FIRA and antisolvent) in the topographic depressions, i.e., at the grain and interdomain boundaries, rather than forming thin films that cover the entire samples.

It has also been suggested that the phase difference observed at grain boundaries is caused by a local dissolution due to the cholorobenzene solvent rather than a PMMA deposit. To rule out this possibility, Figure S7 shows AFM scans of two sections of the same antisolvent sample, as deposited (Figure S7a,b) and after pure chlorobenzene (no PMMA) spin-coating (Figure $S 7 c, d$ ). These results indicate that there is no significant change in roughness nor in phase caused by the solvent wash. Moreover, both phase analyses show an almost flat sample with very low variances across the entire scans, very far from the $\sim 50^{\circ}$ difference of Figures $3 \mathrm{~d}$ and $3 \mathrm{~h}$. Complementary information on the PMMA distribution can be obtained from electron microscopy, particularly from backscattered electrons (Figures S8 and S9).

Solar cells were produced from perovskite films, with and without PMMA treatment. Figure $4 \mathrm{a}$ and Table 1 show the main photovoltaic parameters for the AS and FIRA, reference, and PMMA-treated devices. Antisolvent devices yielded better average results, with higher average open-circuit voltages $V_{\text {oc }}$ short-circuit current densities $J_{s c}$ and fill factors FF, resulting in higher power conversion efficiencies (PCE) than FIRA annealed devices. For the AS devices, the PMMA treatment does not provide a significant increase in $V_{\text {oc }}$ with an average gain of just over $2 \mathrm{mV}$, while $J_{\mathrm{sc}}$ remains unchanged upon
PMMA treatment. In FIRA devices, the polymer treatment does not result in any $V_{\mathrm{oc}}$ increment but only an increase in $J_{\mathrm{sc}}$ by $0.1 \mathrm{~mA} \mathrm{~cm}{ }^{-2}$. While the effect of PMMA treatment on $V_{\text {oc }}$ and $J_{\mathrm{sc}}$ is rather minor, the fill factor increases by more than $1 \%$ for FIRA devices and exceeds $4 \%$ for AS devices. This improvement in fill factor translates into an average PCE increase by $0.4 \%$ and more than $1 \%$ for FIRA and AS cells, respectively.

Figure $4 b, c$ show the $J-V$ curves for the AS and FIRA champion devices, respectively. $V_{\text {oc }}$ and $J_{\text {sc }}$ have similar values in Figure $4 b$, but there is a clear increase in fill factor for the PMMA-treated device, resulting in an efficiency increase from 19.4 to $20.4 \%$. The insets show the stabilized power output (SPO) for both devices. The PMMA antisolvent-treated sample presents a stabilized output of $20 \%$, significantly higher than the nontreated sample. For the FIRA annealed devices in Figure $4 c$, the champion PMMA-treated device has a slightly higher efficiency than the best reference cell, mainly due to a higher $V_{\text {oc }}$ rather than an increase in $J_{\text {sc }}$ and FF. The SPO in the inset in Figure 4c reveals a lower efficiency than antisolvent devices, but in contrast to AS cells, both FIRA devices have remarkably higher stability, with an almost negligible loss in performance. Finally, FIRA devices have a somewhat higher hysteresis than antisolvent cells, which is in agreement with the overall higher efficiency of antisolvent samples.

Dark $J-V$ measurements have been performed (see Figure S10a,b); the results suggest a slight effect in series and shunt resistance for the AS samples, while for the FIRA samples, 
there are no significant differences. Long-term stability analysis of unencapsulated devices, performed after 7 months of sample storage in the dark and dry air, is shown in Figure S10c. Even though there have been some data acquisition interruptions during the analysis, PMMA results in a small beneficial effect on both antisolvent and FIRA devices.

Finally, about the morphological and electrical properties of PMMA films, ellipsometric measurements show that the thickness of the deposited layers is in the range from $0.5 \mathrm{~nm}$ for a solution concentration of $0.1 \mathrm{mg} / \mathrm{mL}$ and approaches 19 $\mathrm{nm}$ for highly concentrated solutions of $10 \mathrm{mg} / \mathrm{mL}$ (Figure S11a-c). The resistance increment due to a conformal and smooth layer as that deposited on polished silicon is much more significant, increasing by factors of 5 to 20 times the original resistance value (Figure S11d) than when deposited on a moderately rough surface as ITO-covered glass (Figure S11e), where the resistance only increases by a factor of 2 . ITO roughness is approximately in the same order of AS perovskite; $^{25}$ therefore, as in the previous analyses (Figure 4), PMMA does not form a continuous film on the ITO surface but rather accumulates in the topographic depressions and affects the substrate conductivity to a much smaller extent. This phenomenon is further analyzed in (Figure S12), where it is possible to appreciate how the increasing concentration of the PMMA solution results in a gradual planarizing effect.

The PL analysis has shown that PMMA deposition onto the perovskite layers does not result in an increment in carrier lifetime or PL intensity. It does not result neither in a noticeable $V_{\text {oc }}$ increment of devices. These phenomena are usually attributed to the passivation of point surface defects, ${ }^{15,18}$ suggesting a disagreement with the hypothesis of a chemical passivation mechanism. The AFM analysis in Figures 3 and 4 show that the PMMA does not form a conformal surface layer on the perovskite but rather accumulates into topographic depressions, grain boundaries, and crystal defects.

Table S1 summarizes the parameters of the champion devices from the literature. The $V_{\text {oc }}$ improvements range from a negligible increment (this work) to $50 \mathrm{mV}$, with most common values of $20-30 \mathrm{mV}$. On the other hand, FF increases from $4^{26}$ to $10 \%,{ }^{22}$ making the FF increase the most noticeable effect of the use of PMMA interlayers and the main responsible for the improvement of device performance.

In this context, it is useful to examine the well-known effect of the shunt resistance $R_{\mathrm{sh}}$ on the fill factor, ${ }^{32}$ given by

$$
\mathrm{FF}_{\mathrm{sh}}=\mathrm{FF}_{0}\left(1-\frac{V_{\mathrm{oc}}}{R_{\mathrm{sh}} I_{\mathrm{sc}}}\right)
$$

with $\mathrm{FF}_{0}$ being the ideal fill factor in the absence of resistive losses. More information about the photovoltaic cell model is given in the Supporting Information. Table 1 lists the average values of shunt resistance obtained by analyzing the device data of this study, reporting values that are among the highest in perovskite solar cells, ${ }^{3,34}$ PMMA treatment of AS perovskite layers resulted in an increase in the average $R_{\mathrm{sh}}$ by more than $30 \%$, giving rise to the fill factor increase and thereby to the improvement in cell efficiency. In FIRA devices, the $R_{\mathrm{sh}}$ increase and its effect on fill factor and cell efficiency is less pronounced.

Note that the effect of the PMMA treatment of PSCs is similar to the edge isolation in silicon solar cells. One of the last steps in Si cell production consists of the isolation of the cell edges, where the $\mathrm{p}$ - and $\mathrm{n}$-doped parts of the device may be in contact, by laser engraving or plasma etching the borders. ${ }^{35}$ These steps increase the shunt resistance and fill factor, accompanied by minor increases in $V_{\text {oc }}$ and $J_{\text {sc }}$ (eqs S1 and S2). $V_{\text {oc }}$ and $J_{\text {sc }}$ improvements are also present in PSCs in addition to the most noticeable fill factor improvement. $^{21,22,25,26,36}$

Summarizing our observations, spin-coating a very lowconcentration PMMA solution onto perovskite layers increases the shunt resistance and the fill factor. The deposited PMMA acts exclusively as a very good electrical isolator, for which it is very well-known. ${ }^{37}$ By accumulation at perovskite grain boundaries and defects, it physically prevents charge carriers from reaching these locations, rather than chemically passivating electronic trap states that may arise from surface defects. This isolation of the grain borders is particularly important, as it was recently shown that some of these have particularly high carrier mobilities ${ }^{38}$ and consequently offer a preferential path for shunting (Figure 5a,c). Furthermore, by

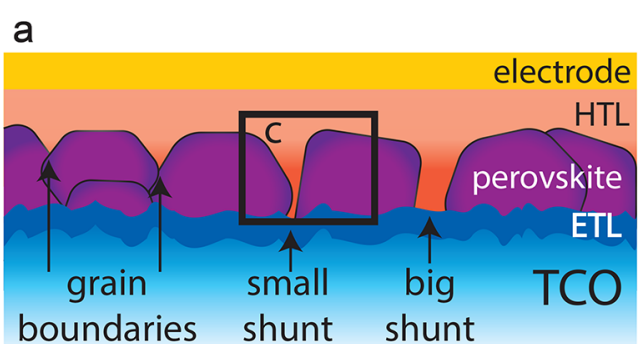

b

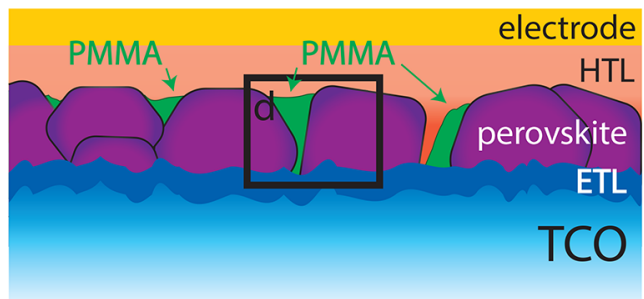

C
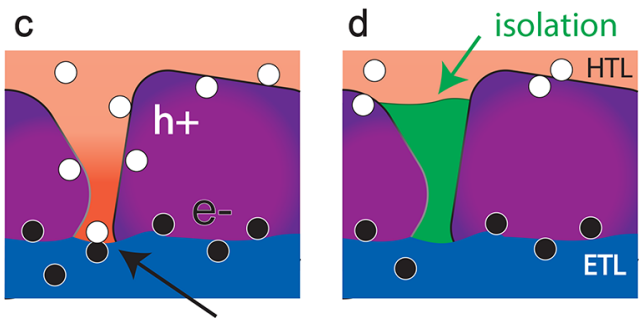

recombination

Figure 5. Schemes of PSC cross sections (a) without and (b) with PMMA treatment. (c,d) Schemes of carrier transport shunting and its prevention in the absence and presence of PMMA, respectively.

PMMA accumulation in pinholes and areas not covered by the perovskite layer, a physical contact between the HTL and ETL is prevented (Figure 5b,d), significantly reducing device shunting. Overall, physical isolation rather than chemical passivation effects benefit device performance, explaining the results described above and those reported in earlier publications. $^{21,22,25,26,36,39}$

The amount of PMMA required to effectively isolate all defects in a device will depend on the compactness of the perovskite layer, the pinhole density, and their size. Less 
compact films with bigger pinholes, like those appearing in FIRA devices, require higher amounts of PMMA, explaining why the FIRA devices described above benefit less from the PMMA treatment. Given that the uncovered substrate areas are quite large (Figures 1, 3, and S8), they may require higher PMMA concentrations than those used in the present study. On the other hand, AS devices feature very compact perovskite layers and are less prone to pinholes, requiring only small amounts of PMMA $(0.1 \mathrm{mg} / \mathrm{mL})$ for their effective isolation.

Note that the introduction of an insulating interlayer may also increase series resistance, as seen in Figure S10, reducing the device current (eq S1), particularly when a conformal insulating layer separates the perovskite from adjacent charge collecting layers. The trade-off between the beneficial and detrimental effects of increasing the shunt and the series resistance, respectively, must be carefully managed by adjusting the deposition conditions for each device manufacture protocol. The use of higher concentrations of polymer could potentially lead to more surface coverage and an effective passivation of surface defects; however at the expense of increasing the series resistance, and upon further increasing the PMMA film thickness device efficiencies drop dramatically. ${ }^{26,36}$

The physical isolation mechanism should apply to any perovskite composition, to $\mathrm{n}-\mathrm{i}-\mathrm{p}$ or $\mathrm{p}-\mathrm{i}-\mathrm{n}$ architectures, and to mesoporous and planar electrode morphologies. Showing a positive effect for the diverse perovskite morphologies generated by the AS and FIRA protocols confirms the broad applicability of the process. The use of a very low polymer solution concentration provides an effective shunting isolation without increasing the series resistance. Finally, since the perovskite remains in immediate contact with the surrounding carrier transport layers, this method can be used in combination with other surface passivation methods, which could be applied before or after the PMMA treatment.

\section{ASSOCIATED CONTENT}

\section{SI Supporting Information}

The Supporting Information is available free of charge at https://pubs.acs.org/doi/10.1021/acsenergylett.1c01187.

Experimental procedures for device fabrication and characterization, photovoltaic model, device structure, additional TRPL data, SEM and AFM images, ellipsometric and $J-V$ analysis of PMMA films, dark $J-V$ measurements, long-term stability of devices, and results from previous works found in bibliography (PDF)

\section{AUTHOR INFORMATION}

\section{Corresponding Authors}

Efrain Ochoa-Martinez - Adolphe Merkle Institute, CH-1700 Fribourg, Switzerland; 이이. orcid.org/0000-0002-5565-0954; Phone: +41 26300 8960; Email: efrain.ochoamartinez@ unifr.ch

Michael Saliba - Institute for Photovoltaics (ipv), 70569 Stuttgart, Germany; Helmholtz Young Investigator Group FRONTRUNNER, IEKS Photovoltaics, Forschungszentrum

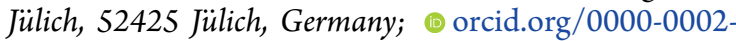
6818-9781; Phone: +49 (0)711 685-67140; Email: michael.saliba@ipv.uni-stuttgart.de

\section{Authors}

Mario Ochoa - Laboratory for Thin Films and Photovoltaics, Empa-Swiss Federal Laboratories for Materials Science and Technology, CH-8600 Duebendorf, Switzerland

Roberto D. Ortuso - Adolphe Merkle Institute, $\mathrm{CH}-1700$ Fribourg, Switzerland

Parnian Ferdowsi - Adolphe Merkle Institute, CH-1700 Fribourg, Switzerland

Romain Carron - Laboratory for Thin Films and Photovoltaics, Empa-Swiss Federal Laboratories for Materials Science and Technology, CH-8600 Duebendorf, Switzerland

Ayodhya N. Tiwari - Laboratory for Thin Films and Photovoltaics, Empa-Swiss Federal Laboratories for Materials Science and Technology, CH-8600 Duebendorf, Switzerland

Ullrich Steiner - Adolphe Merkle Institute, $\mathrm{CH}-1700$

Fribourg, Switzerland; o orcid.org/0000-0001-5936-339X

Complete contact information is available at:

https://pubs.acs.org/10.1021/acsenergylett.1c01187

\section{Notes}

The authors declare no competing financial interest.

Open Data statement: Original data are deposited and freely available in Zenodo repository at https://zenodo.org/record/ 5033465.

\section{ACKNOWLEDGMENTS}

This work was partially funded by a Swiss Government Excellence Scholarship (2017.1080) and by the European Union's Horizon 2020 Research and Innovation Programme under the Marie Sklodowska-Curie project PerSisTanCe with grant agreement No. 841005 (E.O.M.). It also received financial support from the Swiss State Secretary for Education, Research and Innovation (SERI) under contract number 17.00105 (EMPIR project HyMet). The EMPIR programme is cofinanced by the Participating States and by the European Union's Horizon 2020 research and innovation programme. E.O.M., R.D.O., P.F., and U.S. acknowledge financial support by the Adolphe Merkle Foundation. E.O.M.would like to thank Dr. Silver Hamill Turren-Cruz from Helmholtz-Zentrum Berlin for fruitful discussions and Dr. Jovana Milic from the University of Fribourg and Brian Carlsen from the Laboratory of Photomolecular Science (EPFL) for facilitating and carrying out stability measurements.

\section{REFERENCES}

(1) IEA. World Energy Balances (database). 2020. https://www.iea. org/reports/world-energy-balances-overview, Accessed: 2021-0517.

(2) IEA. Solar PV (Tracking report). 2020. https://www.iea.org/ reports/solar-pv, Accessed: 2021-05-17.

(3) IEA. Renewable Energy Market Update, Outlook for 2020 and 2021. 2020. https://www.iea.org/reports/renewable-energy-marketupdate, Accessed: 2021-05-17.

(4) Fraunhofer Insitute for Solar Energy Systems. Photovoltaics Report. 2020. https://www.ise.fraunhofer.de/content/dam/ise/de/ documents/publications/studies/Photovoltaics-Report.pdf, Accessed: 2021-05-17.

(5) Green, M. A.; Dunlop, E. D.; Hohl-Ebinger, J.; Yoshita, M.; Kopidakis, N.; Ho-Baillie, A. W. Y. Solar cell efficiency tables (Version 55). Prog. Photovoltaics 2020, 28, 3-15.

(6) Li, J.; Munir, R.; Fan, Y.; Niu, T.; Liu, Y.; Zhong, Y.; Yang, Z.; Tian, Y.; Liu, B.; Sun, J.; Smilgies, D.-M.; Thoroddsen, S.; Amassian, A.; Zhao, K.; Liu, S. Phase Transition Control for High-Performance Blade-Coated Perovskite Solar Cells. Joule 2018, 2, 1313-1330. 
(7) Ma, Q.; Huang, S.; Wen, X.; Green, M. A.; Ho-Baillie, A. W. Y. Hole Transport Layer Free Inorganic Cs $\mathrm{PbIBr}_{2}$ Perovskite Solar Cell by Dual Source Thermal Evaporation. Adv. Energy Mater. 2016, 6, 1502202 .

(8) Sánchez, S.; Hua, X.; Phung, N.; Steiner, U.; Abate, A. Flash Infrared Annealing for Antisolvent-Free Highly Efficient Perovskite Solar Cells. Adv. Energy Mater. 2018, 8, 1702915.

(9) Sánchez, S.; Steiner, U.; Hua, X. Phase Evolution During Perovskite Formation-Insight from Pair Distribution Function Analysis. Chem. Mater. 2019, 31, 3498-3506.

(10) Muscarella, L. A.; Hutter, E. M.; Sanchez, S.; Dieleman, C. D.; Savenije, T. J.; Hagfeldt, A.; Saliba, M.; Ehrler, B. Crystal Orientation and Grain Size: Do They Determine Optoelectronic Properties of $\mathrm{MAPbI}_{3}$ Perovskite? J. Phys. Chem. Lett. 2019, 10, 6010-6018.

(11) Descoeudres, A.; Holman, Z. C.; Barraud, L.; Morel, S.; De Wolf, S.; Ballif, C. > 21\% Efficient Silicon Heterojunction Solar Cells on $\mathrm{n}$ - and $\mathrm{p}$-Type Wafers Compared. IEEE Journal of Photovoltaics 2013, 3, 83-89.

(12) Chantana, J.; Kawano, Y.; Nishimura, T.; Kato, T.; Sugimoto, H.; Minemoto, T. Characterization of Cd-Free $\mathrm{Zn}_{1-x} \mathrm{Mg}_{x} \mathrm{xO}: \mathrm{Al} /$ $\mathrm{Zn}_{1-x} \mathrm{Mg}_{x} \mathrm{O} / \mathrm{Cu}(\mathrm{In}, \mathrm{Ga})(\mathrm{S}, \mathrm{Se})_{2}$ Solar Cells Fabricated by an All Dry Process Using Ultraviolet Light Excited Time-Resolved Photoluminescence. ACS Appl. Mater. Interfaces 2019, 11, 7539-7545.

(13) Chen, B.; Rudd, P. N.; Yang, S.; Yuan, Y.; Huang, J. Imperfections and their passivation in halide perovskite solar cells. Chem. Soc. Rev. 2019, 48, 3842-3867.

(14) Jiang, Q.; Zhao, Y.; Zhang, X.; Yang, X.; Chen, Y.; Chu, Z.; Ye, Q.; Li, X.; Yin, Z.; You, J. Surface passivation of perovskite film for efficient solar cells. Nat. Photonics 2019, 13, 460-466.

(15) Lee, S.-H.; Jeong, S.; Seo, S.; Shin, H.; Ma, C.; Park, N.-G. Acid Dissociation Constant: A Criterion for Selecting Passivation Agents in Perovskite Solar Cells. ACS Energy Letters 2021, 6, 1612-1621.

(16) Kanwat, A.; Yantara, N.; Ng, Y. F.; Hooper, T. J. N.; Rana, P. J. S.; Febriansyah, B.; Harikesh, P. C.; Salim, T.; Vashishtha, P.; Mhaisalkar, S. G.; Mathews, N. Stabilizing the Electroluminescence of Halide Perovskites with Potassium Passivation. ACS Energy Letters 2020, 5, 1804-1813.

(17) Schulze, P. S. C.; Bett, A. J.; Bivour, M.; Caprioglio, P.; Gerspacher, F. M.; Kabakl1, O. S.; Richter, A.; Stolterfoht, M.; Zhang, Q.; Neher, D.; Hermle, M.; Hillebrecht, H.; Glunz, S. W.; Goldschmidt, J. C. 25.1 High-Efficiency Monolithic Perovskite Silicon Tandem Solar Cell with a High Bandgap Perovskite Absorber. Solar RRL 2020, 4, 2000152.

(18) Noel, N. K.; Abate, A.; Stranks, S. D.; Parrott, E. S.; Burlakov, V. M.; Goriely, A.; Snaith, H. J. Enhanced Photoluminescence and Solar Cell Performance via Lewis Base Passivation of OrganicInorganic Lead Halide Perovskites. ACS Nano 2014, 8, 9815-9821.

(19) Bi, D.; Yi, C.; Luo, J.; Décoppet, J.-D.; Zhang, F.; Zakeeruddin, S. M.; Li, X.; Hagfeldt, A.; Grätzel, M. Polymer-templated nucleation and crystal growth of perovskite films for solar cells with efficiency greater than 21\%. Nature Energy 2016, 1, 16142.

(20) Chen, K.; Hu, Q.; Liu, T.; Zhao, L.; Luo, D.; Wu, J.; Zhang, Y.; Zhang, W.; Liu, F.; Russell, T. P.; Zhu, R.; Gong, Q. Charge-Carrier Balance for Highly Efficient Inverted Planar Heterojunction Perovskite Solar Cells. Adv. Mater. 2016, 28, 10718-10724.

(21) Chen, Y.; Shi, J.; Li, X.; Li, S.; Lv, X.; Sun, X.; Zheng, Y.-Z.; Tao, $\mathrm{X}$. A universal strategy combining interface and grain boundary engineering for negligible hysteresis and high efficiency (21.41\%) planar perovskite solar cells. J. Mater. Chem. A 2020, 8, 6349-6359. (22) Bi, S.; Zhang, X.; Qin, L.; Wang, R.; Zhou, J.; Leng, X.; Qiu, X.; Zhang, Y.; Zhou, H.; Tang, Z. Incorporating an Inert Polymer into the Interlayer Passivates Surface Defects in Methylammonium Lead Halide Perovskite Solar Cells. Chem. - Eur. J. 2017, 23, 14650-14657.

(23) Peng, J.; et al. Interface passivation using ultrathin polymerfullerene films for high-efficiency perovskite solar cells with negligible hysteresis. Energy Environ. Sci. 2017, 10, 1792-1800.

(24) Ferdowsi, P.; Ochoa-Martinez, E.; Alonso, S. S.; Steiner, U.; Saliba, M. Ultrathin polymeric films for interfacial passivation in wide band-gap perovskite solar cells. Sci. Rep. 2020, 10, 22260.
(25) Wang, F.; Shimazaki, A.; Yang, F.; Kanahashi, K.; Matsuki, K.; Miyauchi, Y.; Takenobu, T.; Wakamiya, A.; Murata, Y.; Matsuda, K. Highly Efficient and Stable Perovskite Solar Cells by Interfacial Engineering Using Solution-Processed Polymer Layer. J. Phys. Chem. C 2017, 121, 1562-1568.

(26) Liu, P.; Liu, Z.; Qin, C.; He, T.; Li, B.; Ma, L.; Shaheen, K.; Yang, J.; Yang, H.; Liu, H.; Liu, K.; Yuan, M. High-performance perovskite solar cells based on passivating interfacial and intergranular defects. Sol. Energy Mater. Sol. Cells 2020, 212, 110555.

(27) Günzler, A.; Bermúdez-Ureña, E.; Muscarella, L. A.; Ochoa, M.; Ochoa-Martínez, E.; Ehrler, B.; Saliba, M.; Steiner, U. Shaping Perovskites: In Situ Crystallization Mechanism of Rapid Thermally Annealed, Prepatterned Perovskite Films. ACS Appl. Mater. Interfaces 2021, 13, 6854-6863.

(28) Ghahremani, A. H.; Pishgar, S.; Bahadur, J.; Druffel, T. Intense Pulse Light Annealing of Perovskite Photovoltaics Using Gradient Flashes. ACS Applied Energy Materials 2020, 3, 11641-11654.

(29) Sánchez, S.; Jerónimo-Rendon, J.; Saliba, M.; Hagfeldt, A. Highly efficient and rapid manufactured perovskite solar cells via Flash InfraRed Annealing. Mater. Today 2020, 35, 9-15.

(30) Krogmeier, B.; Staub, F.; Grabowski, D.; Rau, U.; Kirchartz, T. Quantitative analysis of the transient photoluminescence of $\mathrm{CH}_{3} \mathrm{NH}_{3} \mathrm{PbI}_{3} / \mathrm{PC}_{61} \mathrm{BM}$ heterojunctions by numerical simulations. Sustainable Energy \& Fuels 2018, 2, 1027-1034.

(31) García, R. Phase Imaging Atomic Force Microscopy. Amplitude Modulation Atomic Force Microscopy 2010, 91-101.

(32) Hilali, M. M.; Nakayashiki, K.; Ebong, A.; Rohatgi, A. Highefficiency (19\%) screen-printed textured cells on low-resistivity floatzone silicon with high sheet-resistance emitters. Prog. Photovoltaics 2006, 14, 135-144.

(33) Singh, R.; Sandhu, S.; Lee, J.-J. Elucidating the effect of shunt losses on the performance of mesoporous perovskite solar cells. Sol. Energy 2019, 193, 956-961.

(34) Liao, P.; Zhao, X.; Li, G.; Shen, Y.; Wang, M. A New Method for Fitting Current-Voltage Curves of Planar Heterojunction Perovskite Solar Cells. Nano-Micro Lett. 2018, 10, 5.

(35) Booth, H. Laser processing in industrial solar module manufacturing. J. Laser Micro/Nanoeng. 2010, 5, 183-191.

(36) Wang, Q.; Dong, Q.; Li, T.; Gruverman, A.; Huang, J. Thin Insulating Tunneling Contacts for Efficient and Water-Resistant Perovskite Solar Cells. Adv. Mater. 2016, 28, 6734-6739.

(37) Kim, T. G.; Jeong, E. H.; Lim, S. C.; Kim, S. H.; Kim, G. H.; Kim, S. H.; Jeon, H.-Y.; Youk, J. H. PMMA-based patternable gate insulators for organic thin-film transistors. Synth. Met. 2009, 159, $749-753$.

(38) Song, J.; Zhou, Y.; Padture, N. P.; Huey, B. D. Anomalous 3D nanoscale photoconduction in hybrid perovskite semiconductors revealed by tomographic atomic force microscopy. Nat. Commun. 2020, 11, 3308.

(39) Peng, J.; et al. A Universal Double-Side Passivation for High Open-Circuit Voltage in Perovskite Solar Cells: Role of Carbonyl Groups in Poly(methyl methacrylate). Adv. Energy Mater. 2018, 8, 1801208. 